\title{
Protocol of a study investigating breath-hold techniques for upper-abdominal radiation therapy (BURDIE): addressing the challenge of a moving target
}

\author{
Briana Farrugia ${ }^{1,2^{*}}$, Richard Khor ${ }^{1}$, Farshad Foroudi ${ }^{1}$, Michael Chao ${ }^{1}$, Kellie Knight ${ }^{2}$ and Caroline Wright $^{2}$
}

\begin{abstract}
Background: Radiation therapy to upper abdominal sites is technically challenging due to motion of tumors and surrounding organs resulting from normal respiration. Breath-hold, using an Active Breathing Coordinator is one strategy used to reduce motion in these tumor sites. Though widely used, no studies have prospectively compared the different breath-hold techniques (inspiration, deep-inspiration and expiration) using ABC in the same patient cohort.

Methods: Patients planned for radiation therapy to upper abdominal tumors are invited to participate in this prospective study. Participants attempt three breath hold techniques: inspiration, deep-inspiration and expiration breathhold, in random order. kV fluoroscopy images of the dome of diaphragm are taken of five consecutive breath-holds in each technique. Reproducibility and stability of tumour position are measured, and used to select the technique with which to proceed to planning and treatment. Reproducibility at planning and each treatment fraction is measured, along with breath hold time, treatment efficiency and patient experience.

Discussion: The screening method was validated after the first three participants. This screening process may be able to select the best breath-hold technique for an individual, which may lead to improved reproducibility. The screening process is being piloted as a prospective clinical trial.

Trial registration: Australian New Zealand Clinical Trials Registry (ANZCTR): 12618001691235. Registered 12th October 2018. https://www.anzctr.org.au/Trial/Registration/TrialReview.aspx?id=376109\&isReview=true.
\end{abstract}

Keywords: Breath holding, Neoplasms, Radiotherapy

\section{Background}

Radiation therapy (RT) to upper abdominal (UA) sites, including liver, pancreas, kidneys and adrenal glands, is technically challenging. This is due to the proximity of the tumor to organs at risk (OAR), and OAR motion due to both respiration and physiological variation, such as filling of gastro-intestinal organs [1].

*Correspondence: briana.farrugia@austin.org.au

${ }^{1}$ Radiation Oncology, Olivia Newton-John Cancer Wellness and Research Centre, Austin Health, PO Box 5555, Heidelberg, VIC 3084, Australia

Full list of author information is available at the end of the article
Breath-hold $(\mathrm{BH})$ techniques, either voluntary or assisted, have been implemented to minimize respiratory-induced motion [2-7]. Inspiration Breath-Hold (IBH), Deep-Inspiration Breath-Hold (DIBH) and Expiration Breath-Hold (EBH) are reported in the literature [3-7]. Employing a voluntary IBH technique has dem-

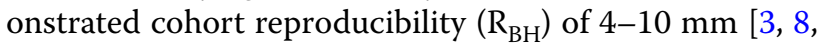
9], whilst voluntary EBH has demonstrated cohort $\mathrm{R}_{\mathrm{BH}}$ of 2-5 mm [3, 9]. When an Active Breathing Coordinator $(\mathrm{ABC})^{\mathrm{TM}}$ device (Elekta, Stockholm, Sweden) is used to assist breath-hold, improvements in $\mathrm{R}_{\mathrm{BH}}$ have been seen, with DIBH intra-fraction cohort $\mathrm{R}_{\mathrm{BH}}$ of $1.3-1.6 \mathrm{~mm}[6$, original author(s) and the source, provide a link to the Creative Commons licence, and indicate if changes were made. The images or other third party material in this article are included in the article's Creative Commons licence, unless indicated otherwise in a credit line to the material. If material is not included in the article's Creative Commons licence and your intended use is not permitted by statutory regulation or exceeds the permitted use, you will need to obtain permission directly from the copyright holder. To view a copy of this licence, visit http://creativecommons.org/licenses/by/4.0/. The Creative Commons Public Domain Dedication waiver (http://creativeco mmons.org/publicdomain/zero/1.0/) applies to the data made available in this article, unless otherwise stated in a credit line to the data. 
7], and EBH intra-fraction cohort $\mathrm{R}_{\mathrm{BH}}$ of $1.5 \mathrm{~mm}$ [10]. Although EBH techniques tend to display a better $R_{B H}$ at a cohort level, in many studies the $\mathrm{R}_{\mathrm{BH}}$ ranges for different $\mathrm{BH}$ techniques overlap [3,9]. This suggests that patients may be able to perform multiple breath hold techniques adequately enough for IGRT. Although population level estimates for the average cohort $\mathrm{R}_{\mathrm{BH}}$ and $\mathrm{S}_{\mathrm{BH}}$ have been done, none yet aim to select the best method for each patient.

There is limited literature available to describe patients' experience of BH. A recent study evaluated 150 patients' experiences of voluntary BH using MR-guided RT using an un-validated questionnaire [11]. Considerable difficulty controlling their tumor position in voluntary $\mathrm{BH}$ was reported by $12.5 \%$ of patients [11]. Another study investigated the patient experience of DIBH, in 41 patients receiving breast RT [12]. More than $90 \%$ of participants rated their experience $>8$ on a 10 point Likert-type scale (where $0=$ not at all; $10=$ extremely) for ease, comfort and control of BH using ABC [12]. To our knowledge, no studies have investigated patients' experiences of multiple $\mathrm{BH}$ techniques.

\section{Methods}

\section{Study design and ethics}

The aims of this study are to evaluate the $\mathrm{R}_{\mathrm{BH}}$ and stability $\left(\mathrm{S}_{\mathrm{BH}}\right)$ of tumor position; patient experience; and efficiency of treatment delivery for each $\mathrm{BH}$ technique. This is a prospective, single-institution study of adults, aged over 18, undergoing RT for malignancies of the liver, pancreas, adrenal gland and kidney. Patients suitable for RT, including Stereotactic Ablative Radiotherapy (SABR) techniques are eligible and invited to participate. The study was approved by our institutional Human Research Ethics Committee (HREC 47012). Once identified, eligible participants are provided with written information about the study from their Radiation Oncologist (RO) or the study coordinator. Informed consent is mandated prior to enrolment. Figure 1 provides an overview of study procedures.

\section{Primary outcome measures}

a $\mathrm{R}_{\mathrm{BH}}$ and $\mathrm{S}_{\mathrm{BH}}$ of UA tumors in IBH, DIBH and EBH, measured pre-planning.

b Number of participants screened into each BH technique.

c $R_{B H}$ of UA tumors at planning, in participant's selected technique.

$\mathrm{d}$ Inter-fraction and intra-fraction $\mathrm{R}_{\mathrm{BH}}$ of UA tumors at each treatment fraction, in participant's selected technique.

\section{Secondary outcome measures}

a Treatment efficiency of IBH, DIBH and EBH, in participant's selected technique.

b Patient-reported experience of IBH, DIBH and EBH, measured pre-planning.

\section{Study procedures}

\section{Breath-Hold Assessment}

All participants undergo protocolled education with $A B C$, then an assessment to confirm their ability to $\mathrm{ABC}-\mathrm{BH}$ (Fig. 2a-c: ABC Device). Participants are screened for eligibility in IBH, DIBH and EBH in random order to reduce risk of bias, using a pre-determined block randomization sequence. Eligibility, breath-hold time $\left(\mathrm{T}_{\mathrm{BH}}\right)$ and $\mathrm{ABC}-\mathrm{BH}$ threshold are recorded for each technique.

$T_{B H}$ Time (seconds) that the participant can BH comfortably, at least three times.

$A B C-B H$ Threshold Volume of air (liters) in the participant's lungs at $\mathrm{BH}$ activation. The radiation therapists determine the threshold with the participant, as follows:

EBH 0.0-0.2 L

$I B H$ Peak volume of air during normal, relaxed respiration. Average of three measurements.

DIBH Maximum peak volume of air during voluntary deep inspiration. Threshold is $80 \%$ of average of three measurements.

\section{Pre-planning reproducibility and stability}

To assess $R_{B H}$ of tumor position between consecutive BHs, and $\mathrm{S}_{\mathrm{BH}}$ of tumor position during each $\mathrm{BH}, 5 \mathrm{kV}$ $\mathrm{X}$-ray fluoroscopy images are acquired in each $\mathrm{BH}$ technique on an Elekta ${ }^{\mathrm{TM}}$ linear accelerator, using the $\mathrm{XVI}^{\mathrm{TM}}$ software "MotionView" function. The position of the tumor, or an appropriate surrogate (diaphragm or fiducial marker), is tracked. Each image is acquired anterior-posteriorly (AP) (Fig. 3), for the complete duration of each of the participant's BH's. $\mathrm{R}_{\mathrm{BH}}$ and $\mathrm{S}_{\mathrm{BH}}$ are defined as follows:

$R_{B H}$ Cranio-caudal position of the tumor/surrogate at the beginning of $\mathrm{BH} 1$ is compared to the position at beginning of $\mathrm{BH} 2-5$. Four observations are recorded per technique. The absolute value (ignoring direction) of the cumulative sum of each observation is determined, and then averaged.

$S_{B H}$ Cranio-caudal displacement of the tumor/surrogate, during each $\mathrm{BH}$. Five observations are recorded 
Recruitment

-Patients screened for eligibility against inclusion/exclusion criteria -Written informed consent provided

\section{Patient Education}

-Participants provided with written information on $A B C$ breath-hold

-30-60 minute coaching session by RTs credentialled in ABC breath hold education

\section{Breath-Hold Assessment}

-Able to breath-hold using $A B C$, including:

- Operate control switch; Tolerate and use mouth-piece and nose-peg

-Breath-hold in at least one technique $>15$ seconds

\section{Pre-Planning Reproducibility \& Stability Assessment}

-Five kV fluoroscopy images in each eligible breath-hold technique (15 images max)

-Reproducibility measurements of tumour/surrogate position from consecutive breath-holds

-Stability measurements of tumour/surrogate position during each breath hold

\section{Participant Experience Questionnaire}

- Qualitative \& quantitative analysis of patient experience

\section{Determination of Treatment Technique}

- Selection of technique according to decision matrix

-Prioritise 1. reproducibility; 2. stability; 3. breath-hold time

\section{Planning Reproducibility}

-Four planning CT scans to assess tumour position reproducibility

\section{Treatment Reproducibility}

-Daily Cone Beam CT (CBCT) images to assess inter-fraction \& intra-fraction reproducibility

Treatment Efficiency

-Determine efficiency of delivery accounting for breath-hold and recovery time

Fig. 1 Study schema

per technique. The mean of the absolute value (ignoring direction) of each observation is then calculated.

\section{Patient experience questionnaire}

Participants are invited to complete a questionnaire (Additional file 1: Appendix 1) to evaluate their experience of each technique. The questionnaire, developed specifically for this study, as there was no available validated questionnaire, includes both quantitative questions using a Likert-type scale; and qualitative open-ended questions, allowing the participant to elaborate on their experience. Participants are asked to rank the techniques in order of their preference. As required, a staff member involved in the breathing and/or reproducibility and stability assessment will conduct a semi-structured interview with the patient to elicit responses to all applicable questions.

\section{Determination of treatment technique}

Following the ABC-BH and pre-planning $\mathrm{R}_{\mathrm{BH}}$ and $\mathrm{S}_{\mathrm{BH}}$ assessments, selection of treatment technique is made according to the decision matrix (Fig. 4), with $R_{B H}$ prioritized. If two or more techniques have equal (to nearest $\mathrm{mm}$ ) mean $\mathrm{R}_{\mathrm{BH}}$, then the technique with better $\mathrm{S}_{\mathrm{BH}}$ is selected. A technique with mean $\mathrm{R}_{\mathrm{BH}}$ and/or $\mathrm{S}_{\mathrm{BH}}>5 \mathrm{~mm}$ will proceed to free-breathing. 

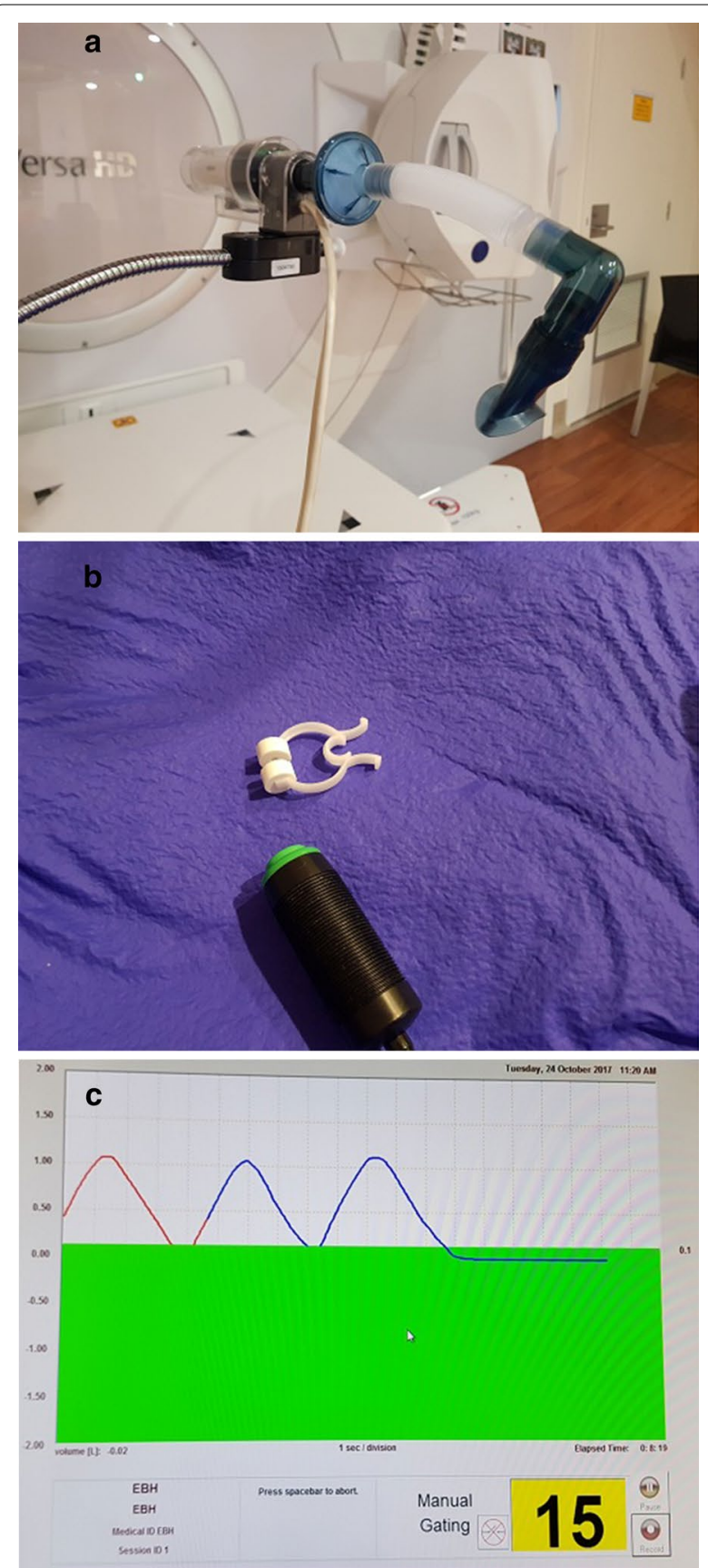

Fig. 2 Active Breathing Coordinator ${ }^{\mathrm{TM}}$ components. a Mouth-piece and spirometer; $\mathbf{b}$ nose-peg and control switch; c operators screen displaying activated EBH indicated by green bar

\section{Planning reproducibility}

Participants have all subsequent planning and treatment using the chosen $\mathrm{BH}$ technique. Four planning computed tomography $(\mathrm{CT})$ scans are acquired for each participant. The scans are acquired sequentially within a $5 \mathrm{~min}$ timeframe, with no patient-repositioning between scans,

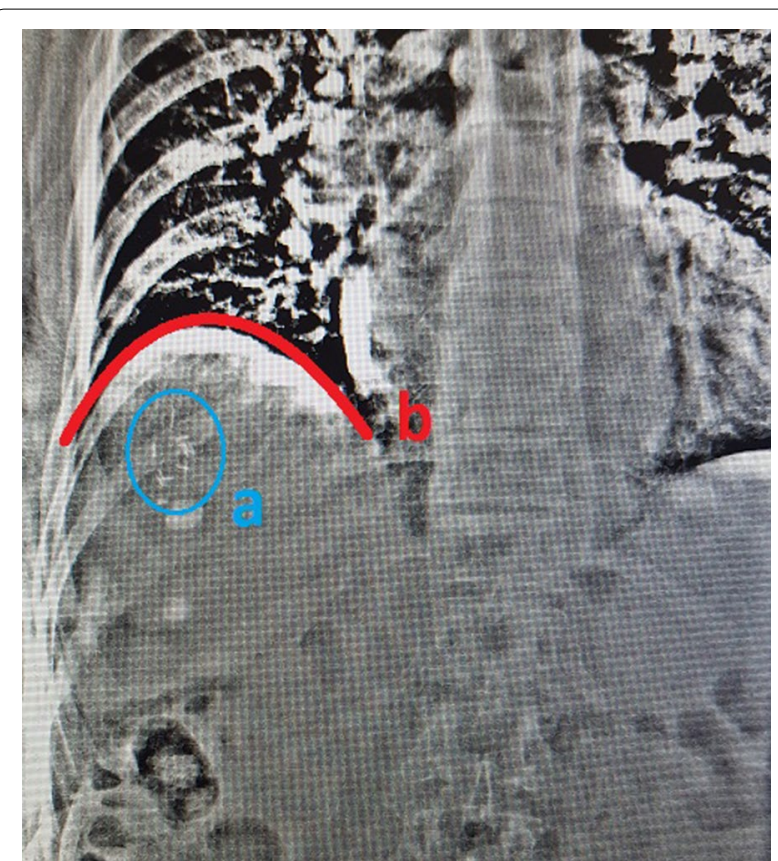

Fig. 3 AP kV fluoroscopy image with fiducial markers (a-blue) and diaphragm position (b-red)

to minimize the impact of repositioning or physiological variation. The Gross Tumor Volume (GTV), tumor surrogates and OARs are contoured on the first planning CT. Post-processing and measurements are completed using a customized workflow in MIM Maestro ${ }^{\circledR}$ software (MIM Software Inc, Cleveland $\mathrm{OH}$ ). The first planning CT is automatically fused to subsequent CTs. Initially, a bone algorithm fusion corrects any gross patient misalignment. Then, a tumor, surrogate or fiducial marker fusion corrects for tumor/organ position. Contours are automatically generated onto each subsequent CT. Resultant contours are reviewed, and manually edited if required to account for deformation. The inter-BH displacement of the tumor and OARs are measured, with bone fusion as the starting position. Planning $\mathrm{R}_{\mathrm{BH}}$ of tumour/surrogate and OARs is defined as follows:

$R_{B H}$ Position, in three planes, of the tumor/surrogate or OAR contour centroid and contour surface in CT1 compared to the position in CT2-4, resulting in three observations. The mean of the absolute values of the cumulative sum of each observation will be calculated.

\section{Treatment reproducibility}

Intra-fraction and inter-fraction $\mathrm{R}_{\mathrm{BH}}$ is measured for each participant. For all treatment fractions, 3D volumetric Cone Beam Computed Tomography (CBCT) 


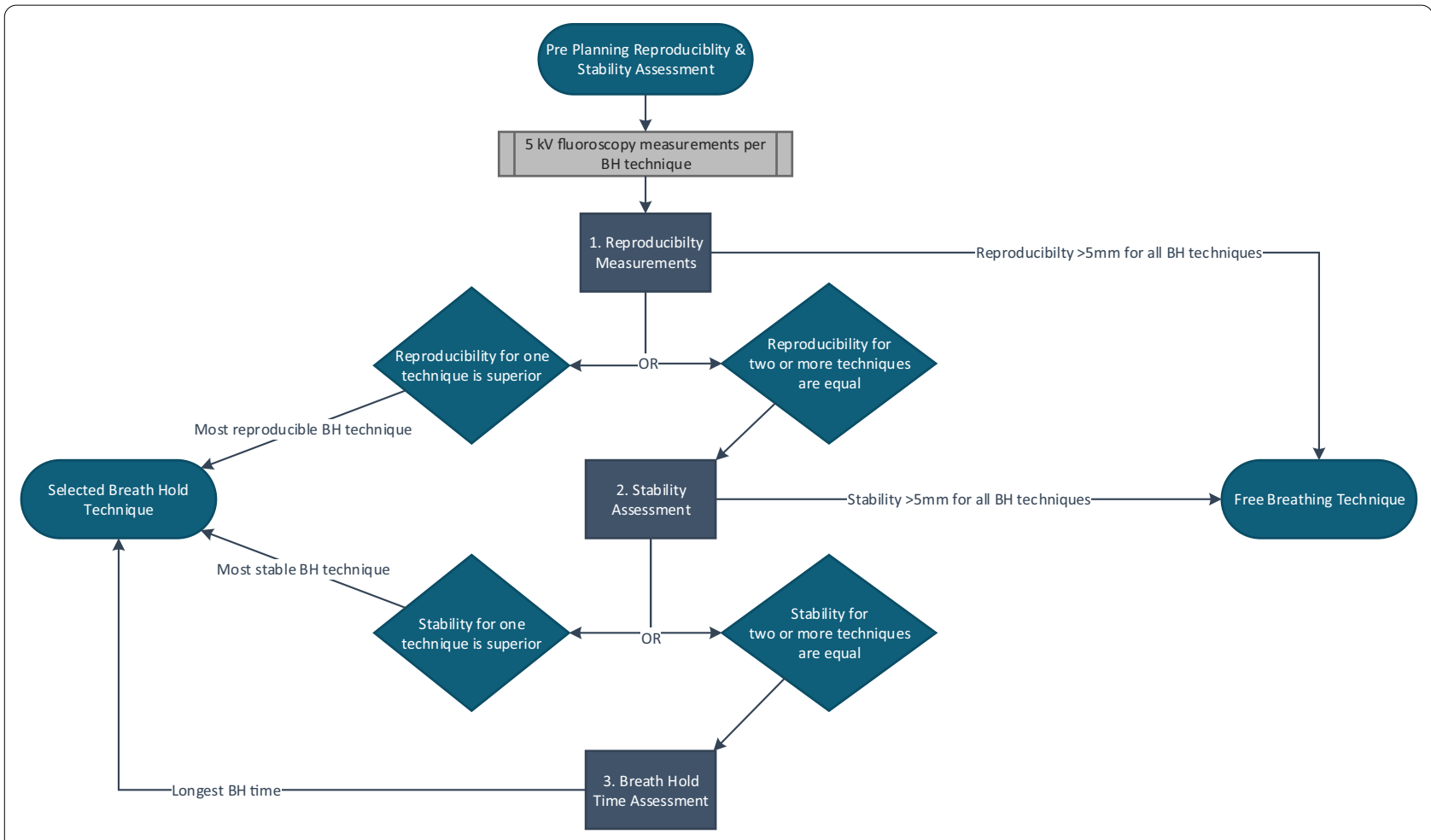

Fig. 4 Decision matrix for selection of treatment technique

images are acquired using Elekta XVI ${ }^{\mathrm{TM}}$ (Elekta, Stockholm, Sweden) before treatment delivery. For participants treated with SABR, post-correction, during, and post-treatment $\mathrm{CBCTs}$ are also acquired. Each CBCT is a $360^{\circ}$ acquisition using several $\mathrm{BHs}$, with acquisition paused between BHs. The CBCT is automatically fused to the planning CT with a bone algorithm to correct patient setup. Then, a soft-tissue fusion corrects for tumor position. Tumor displacement is measured as the correction applied after accounting for patient setup. Treatment $\mathrm{R}_{\mathrm{BH}}$ of tumour is defined as follows:

Inter-Fraction $R_{B H}$ Position, in three planes, of the tumor/surrogate on planning CT compared to the position on CBCT1, at each treatment fraction. Results will be collated for all fractions, and averaged.

Intra-Fraction $R_{B H}$ For those participants with multiple CBCTs per fraction, the position, in three planes, of the tumor/surrogate on CBCT1 compared to the position on each subsequent $\mathrm{CBCT}$, at each treatment fraction. The mean values of the absolute value of the cumulative sum of each observation will be calculated.

\section{Treatment efficiency}

Total treatment time, $\mathrm{T}_{\mathrm{BH}}$, and number of $\mathrm{BHs}$ required to deliver treatment are recorded for the first three fractions for all participants. From this, an estimate of treatment efficiency is determined, as the proportion of total treatment time in which delivery occurred.

\section{Statistical analysis}

Sample size calculation indicates required recruitment of 14-27 participants to be powered to detect a $2 \mathrm{~mm}$ variation in reproducibility, assuming standard deviation of $2 \mathrm{~mm}$ or $3 \mathrm{~mm}$ respectively.

The $\mathrm{R}_{\mathrm{BH}}$ and $\mathrm{S}_{\mathrm{BH}}$ measurements during pre-planning will be collated and described for each participant and each $\mathrm{BH}$ method. The number of patients screened into each $\mathrm{BH}$ technique will be reported, along with descriptive statistics such as the mean and standard deviation (SD) for $\mathrm{R}_{\mathrm{BH}}$ and $\mathrm{S}_{\mathrm{BH}}$ in order to compare each technique. Paired $t$ tests will be used to test for differences between the BURDIE-screened $\mathrm{R}_{\mathrm{BH}}$ and $\mathrm{S}_{\mathrm{BH}}$ with our institutional standard technique $\mathrm{EBH}$, including subset analysis of those participants who do not screen into EBH. Further comparisons will be made to compare each $\mathrm{BH}$ technique's mean $R_{\mathrm{BH}}$ and $\mathrm{S}_{\mathrm{BH}}$ with the selected technique 
mean. Mixed effects models will be used to evaluate the multiple observations for each patient, and each technique.

The planning mean $R_{B H}$ measurements will be compared with the pre-planning results for each participant, and allow an assessment of the correlation between the mean $\mathrm{R}_{\mathrm{BH}}$ values at the two time-points. Paired $t$ tests will be conducted to provide an estimate of the $95 \%$ confidence interval in the paired observations with a margin of $\pm 2 \mathrm{~mm}$ considered to reflect similarity and/or an indication of the non-inferiority margin. Bland-Altman plots, with Pitman's test will be prepared to test for any indication of bias in the pre-planning and planning $R_{B H}$ means. Tests for variation across each $\mathrm{BH}$ technique will be conducted, with assessments of the average $R_{B H}$ values for each technique, the study population and comparisons to the pre-planning time-point.

To evaluate treatment $\mathrm{R}_{\mathrm{BH}}$, correlation coefficients will be estimated as fixed-effects, to indicate the consistency between the inter-fraction and intra-fraction observations. The treatment mean $\mathrm{R}_{\mathrm{BH}}$, will be compared to the pre-planning and planning results, and allow an assessment of the correlation between these paired means. The 95\% confidence interval in the paired observations will be compared to a non-inferiority margin of $\pm 2 \mathrm{~mm}$, with Bland-Altman plots and the Pitman's test used to test for any indication of bias in the treatment $\mathrm{R}_{\mathrm{BH}}$ for comparison with pre-planning and planning means. However, it is expected that that the difference between the preplanning, planning and treatment values may reduce over time, and these differences will be assessed descriptively, and compared to the non-inferiority margin of $\pm 2 \mathrm{~mm}$.

\section{Feasibility assessment}

The methodology was validated after the first three recruited participants. All three participants who were eligible and approached for the trial agreed to participate. All three were able to complete the Breath-Hold Assessment and Pre-Planning Reproducibility and Stability Assessment. A summary of these results is presented in Table 1, with IBH being the BURDIE-selected technique for all three participants.

\section{Discussion}

A process to compare and select the optimal $\mathrm{BH}$ technique for each individual patient, using $\mathrm{ABC}$, was developed. This screening process may be able to select the best $\mathrm{BH}$ technique for an individual, which may lead to improved $\mathrm{R}_{\mathrm{BH}}$. The screening process is being piloted as a prospective clinical trial. The results of this study will be disseminated through publication in peer-reviewed journal(s) and/or conference presentations.
Table 1 Results of feasibility assessment $(n=3)$

\begin{tabular}{|c|c|c|c|c|}
\hline \multirow[t]{2}{*}{ Measure } & & \multicolumn{3}{|c|}{$\begin{array}{l}\text { Breath-hold technique mean } \\
\text { (SD) }\end{array}$} \\
\hline & & DIBH & $\mathrm{IBH}$ & EBH \\
\hline \multicolumn{5}{|l|}{ Participant 1} \\
\hline Tumour site & Kidney & & & \\
\hline Age (years) & 32 & & & \\
\hline Gender (M/F) & M & & & \\
\hline ABC threshold $(L)$ & & 1.4 & 0.6 & 0.1 \\
\hline $\mathrm{T}_{\mathrm{BH}}(\mathrm{s})$ & & 16 & 16 & 16 \\
\hline $\mathrm{S}_{B H}(\mathrm{~mm})$ & & $0.4(0.5)$ & $0.8(0.4)$ & $2.0(1.6)$ \\
\hline $\mathrm{R}_{\mathrm{BH}}(\mathrm{mm})$ & & $4.0(2.6)$ & $0.5(0.6)$ & $1.5(1.3)$ \\
\hline Test order & $\mathrm{EBH}, \mathrm{IBH}, \mathrm{DIBH}$ & & & \\
\hline Selected technique & $\mathrm{IBH}$ & & & \\
\hline \multicolumn{5}{|l|}{ Participant 2} \\
\hline Tumour site & Liver & & & \\
\hline Age (years) & 71 & & & \\
\hline Gender (M/F) & M & & & \\
\hline ABC threshold $(L)$ & & 1.2 & 0.9 & 0.1 \\
\hline $\mathrm{T}_{\mathrm{BH}}(\mathrm{s})$ & & 35 & 35 & 30 \\
\hline $\mathrm{S}_{\mathrm{BH}}(\mathrm{mm})$ & & $2.2(1.5)$ & $1.4(0.5)$ & $2.0(2.3)$ \\
\hline $\mathrm{R}_{\mathrm{BH}}(\mathrm{mm})$ & & $1.0(0.0)$ & $0.25(0.5)$ & $0.75(1.0)$ \\
\hline Test order & $\mathrm{DIBH}, \mathrm{IBH}, \mathrm{EBH}$ & & & \\
\hline Selected technique & $\mathrm{IBH}$ & & & \\
\hline \multicolumn{5}{|l|}{ Participant 3} \\
\hline Tumour site & Liver & & & \\
\hline Age (years) & 68 & & & \\
\hline Gender & M & & & \\
\hline ABC threshold (L) & & 0.8 & 0.6 & 0.1 \\
\hline $\mathrm{T}_{\mathrm{BH}}(\mathrm{s})$ & & 20 & 20 & 15 \\
\hline $\mathrm{S}_{\mathrm{BH}}(\mathrm{mm})$ & & $0.6(0.9)$ & $0.6(0.9)$ & $0.8(1.3)$ \\
\hline $\mathrm{R}_{\mathrm{BH}}(\mathrm{mm})$ & & $2.0(2.3)$ & $1.0(0.8)$ & $3.75(1.0)$ \\
\hline Test order & $\mathrm{IBH}, \mathrm{EBH}, \mathrm{DIBH}$ & & & \\
\hline Selected technique & $\mathrm{IBH}$ & & & \\
\hline
\end{tabular}

$D I B H$ deep-inspiration breath-hold, $I B H$ inspiration breath-hold, $E B H$ expiration breath-hold, $T_{B H}$ time (duration) of breath-hold, $R_{B H}$ reproducibility of breathhold, $S_{B H}$ stability of breath-hold

\section{Supplementary information}

Supplementary information accompanies this paper at https://doi. org/10.1186/s13014-020-01688-z.

Additional file 1: Appendix 1. Patient experience questionnaire.

Abbreviations

ABC: Active Breathing Coordinator; BH: Breath-hold; CBCT: Cone-beam computed tomography; CT: Computed tomography; DIBH: Deep-inspiration breath-hold; EBH: Expiration breath-hold; GTV: Gross tumour volume; IBH: Inspiration breath-hold; kV: Kilovoltage; OAR: Organ at risk; RT: Radiation therapy; $\mathrm{R}_{\mathrm{BH}}$ : Reproducibility of breath-hold; RO: Radiation oncologist; $\mathrm{SABR}$ : Stereotactic ablative radiotherapy; $\mathrm{S}_{\mathrm{BH}}$ : Stability of breath-hold; $\mathrm{T}_{\mathrm{BH}}$ : Time of breath-hold; UA: Upper abdominal. 


\section{Acknowledgements}

The authors wish to thank Mark Tacey for support with statistical analysis.

\section{Authors' contributions}

BF drafted and revised this manuscript. BF, RK, MC, FF, KK and CW participated in study design; and revised this manuscript critically. All authors read and approved the final manuscript.

\section{Funding}

This study is being conducted with financial support of a research grant from the Victorian Medical Radiation Practitioner's Education Trust (VMRPET) and the Australian Government's Research Training Program (RTP). The funding sources had no input into the design of the study; the collection, analysis and interpretation of data nor the manuscript writing.

\section{Availability of data and materials}

Not applicable.

\section{Ethics approval and consent to participate}

This study has been approved by Austin Health Human Research Ethics Committee, approval number: HREC/47012/Austin-2018. All participants provide written informed consent prior to enrolment in the study.

\section{Consent for publication}

Not applicable.

\section{Competing interests}

Author RK received fees from Elekta as an invited speaker for the company. Other authors have no competing interests to declare.

\section{Author details}

${ }^{1}$ Radiation Oncology, Olivia Newton-John Cancer Wellness and Research Centre, Austin Health, PO Box 5555, Heidelberg, VIC 3084, Australia. ${ }^{2}$ Department of Medical Imaging and Radiation Sciences, Faculty of Medicine, Nursing and Health Sciences, Monash University, Wellington Rd, Clayton, VIC 3800, Australia.

Received: 2 March 2020 Accepted: 20 October 2020

Published online: 30 October 2020

\section{References}

1. Benedict SH, Yenice KM, Followill D, et al. Stereotactic body radiation therapy: the report of AAPM TG 101. J Med Phys. 2010;37(8):4078-101. https://doi.org/10.1118/1.3438081
2. Chang BK, Timmerman RD. Stereotactic body radiation therapy: a comprehensive review. Am J Clin Oncol. 2007;30(6):637-44. https://doi. org/10.1097/COC.0b013e3180ca7cb1

3. Kimura T, Hirokawa Y, Murakami Y, et al. Reproducibility of organ position using voluntary breath-hold method with spirometer for extracranial stereotactic radiotherapy. Int J Radiat Oncol Biol Phys. 2004;60(4):1307-13. https://doi.org/10.1016/j.jijrobp.2004.07.718

4. Su L, lordachita I, Zhang Y, et al. Feasibility study of ultrasound imaging for stereotactic body radiation therapy with active breathing coordinator in pancreatic cancer. J Appl Clin Med Phys. 2017;18(4):84-96. https://doi. org/10.1002/acm2.12100

5. Yang W, Fraass BA, Reznik R, et al. Adequacy of inhale/exhale breathhold CT based ITV margins and image-guided registration for free-breathing pancreas and liver SBRT. Radiat Oncol. 2014;9(11):1-9. https://doi. org/10.1186/1748-717X-9-11

6. Zhong $R$, Wang J, Jiang $X$, et al. Hypofraction radiotherapy of liver tumor using cone beam computed tomography guidance combined with active breath control by long breath-holding. Radiother Oncol. 2012;104(3):379-85. https://doi.org/10.1016/j.radonc.2011.11.007

7. Zhao JD, Xu ZY, Zhu J, et al. Application of active breathing control in 3-dimensional conformal radiation therapy for hepatocellular carcinoma: the feasibility and benefit. Radiother Oncol. 2008;87(3):439-44. https:// doi.org/10.1016/j.radonc.2007.12.006

8. Lens E, van der Horst A, Versteijne E, Bel A, van Tienhoven G. Considerable pancreatic tumor motion during breath-holding. Acta Oncol. 2016;55(11):1360-8. https://doi.org/10.1080/0284186X.2016.1221532

9. Lens E, Gurney-Champion OJ, Tekelenburg DR, et al. Abdominal organ motion during inhalation and exhalation breath-holds: pancreatic motion at different lung volumes compared. Radiother Oncol. 2016;121(2):26875. https://doi.org/10.1016/j.radonc.2016.09.012

10. Eccles C, Brock KK, Bissonette JP, Hawkins M, Dawson LA. Reproducibility of liver position using Active Breathing Coordinator for liver cancer radiotherapy. Int J Radiat Oncol Biol Phys. 2006;64(3):751-9. https://doi. org/10.1016/j.jijrobp.2005.05.066

11. Tetar S, Bruynzeel A, Bakker R, et al. Patient-reported outcome measurements on the tolerance of magnetic resonance imaging-guided radiation therapy. Cureus. 2018;10(2):e2236. https://doi.org/10.7759/cureus.2236

12. Beaton NR, Watson S, Browne $P$, et al. Deep inspiration breath hold in breast cancer: development and analysis of a patient experience questionnaire. J Med Imaging Radiat Oncol. 2018;62(6):854-60. https://doi. org/10.1111/1754-9485.12795

\section{Publisher's Note}

Springer Nature remains neutral with regard to jurisdictional claims in published maps and institutional affiliations.
Ready to submit your research? Choose BMC and benefit from:

- fast, convenient online submission

- thorough peer review by experienced researchers in your field

- rapid publication on acceptance

- support for research data, including large and complex data types

- gold Open Access which fosters wider collaboration and increased citations

- maximum visibility for your research: over $100 \mathrm{M}$ website views per year

At BMC, research is always in progress.

Learn more biomedcentral.com/submissions 